\title{
CONCEPT OF DEVICE TO INSPECT INTERNAL TECHNICAL CONDITION OF MARINE PIPELINE
}

\author{
Danuta Warnke \\ Gdańsk University of Technology, Poland
}

\begin{abstract}
The article presents a concept of the device to inspect technical condition of the interior of a marine pipeline. The design assumption was that the vehicle would be built for a specific existing pipeline, which is the Nord Stream gas pipeline. Based on the analysis of operating parameters and dimensions of the pipeline, the economic diameter and the medium flow velocity were calculated. The shape of the structure was proposed, and the measuring instruments and materials for manufacturing the vehicle were selected. The problems of energy supply during vehicle operation, and its installation inside the pipeline were solved.
\end{abstract}

Keywords: Nord Stream, marine pipeline, PIG-s inspection

\section{INTRODUCTION}

Pipeline diagnostics has never been an easy task, especially when the diagnosed object is situated at the bottom of the Baltic Sea. Due to different pipe diameters and types of transported media, each pipeline should have its individual inspection vehicle. The goal of the present work was working out a concept of the device to inspect technical condition of the Nord Stream gas pipeline.

The pipelines can be divided into three categories:

a) Raw material pipelineas - for transporting crude oil or gas;

b) Product pipelines - for transporting finished products

to storage and distribution points;

c) Technological pipelines - such as cooling installations in nuclear refineries, for instance.

The Nord Stream pipeline belongs to the first category. Modern pipelines offer the possibility of checking their technical condition from inside, without disconnecting them from service (so-called In Line Inspection). The inspection devices, co-called "smart PIGs", find places on pipe walls which need careful monitoring or repair. Smart pistons are intended to perform two basic functions, which are inspection of pipeline interior and removal of deposits and dirt [1]. The pipeline can be cleaned mechanically or chemically. Here, three types of drives for cleaning devices can be named:

a) mechanical drive (for instance a rope tied to pull the access eye along the pipe),

b) hydraulic drive (medium is used as the drive for smart pistons),

c) pneumatic drive (with compressed air as the driving medium).

The designed device will have hydraulic drive.

In general, external offshore diagnostics can be done by divers, but at larger depths unmanned devices are used which are remotely controlled from the ship. Internal inspection of the pipeline is usually done using probes and smart pistons, also referred to as PIGs [8].

A list of detected inner damages of the pipeline includes:

a) corrosion,

b) material losses,

c) shape changes,

d) cracks. 
A similar list of the detected external pipeline damages includes:

a) corrosion,

b) mechanical damages caused by marine vehicles or animals,

c) human error - material defects, badly prepared foundation.

There are several types of smart pistons used for inspecting inner walls. Their division can base on the used measuring technology:

a) Mechanical devices - which inspect changes of inner geometry and/or mechanical damages;

b) Magnetic devices - making use of magnetic field technology to assess corrosion and cracks;

c) Ultrasound devices - used for measuring pipe wall thickness;

d) Electromagnetic devices - making use of so-called Foucault currents [19].

The designed device belongs to the first group: mechanical inspection devices.

\section{STRUCTURE \\ OF TYPICAL MECHANICAL PIG}

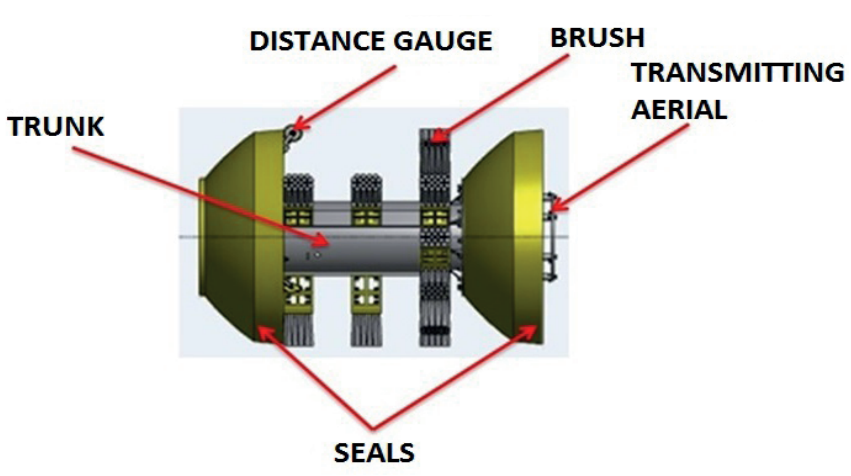

Fig. 1 Structure of typical PIG [18]

The medium flowing in the pipeline drives the device, due to the presence of tightly fitting elements - collars. A large number of cleaning brushes are usually used in oil pipelines, in which more impurities are collected than in gas pipelines [4]. Distance meters are used for determining the current position of the device. The used transmitting antenna makes it possible to localise the PIG in an emergency situation. Searching for the lost device is rather troublesome, as the signal transmitted by the antenna is relatively low. That is why this method works well in ground pipelines. All electronics of the device is placed inside the tight casing, which should also have space for a relatively large battery supplying the deck computer used for recording signals coming from the measuring sensors. The length of the diagnostic devices can reach as much as $4 \mathrm{~m}$. The larger the number of measuring segments, the more precise image of inner pipeline walls is recorded. A longer device is more likely to be wedged inside the pipe, therefore the pipeline is to be cleaned up before inserting the smart piston into it. This can be done with the aid of cleaning PIGs, having the form of a sphere, or a double piston [2]. Their task is to remove methane hydrates created as a result of connection of methane and water molecules. This connection of molecules is facilitated by low temperature and high-pressure which occur in each marine pipeline. The creation of hydrated decreases the transporting cross-section. Another method to eliminate methane hydrates is removal of water from the transported medium, or adding methanol or ethylene glycol to the medium. The amounts of hydrate deposits are to be monitored and removed at least once a year. Attention should also be paid to the starting and finishing stations used for inserting the vehicle in the pipeline and removing it. A standard scheme of inserting the device in the pipeline is shown in Fig. 2 and described below:

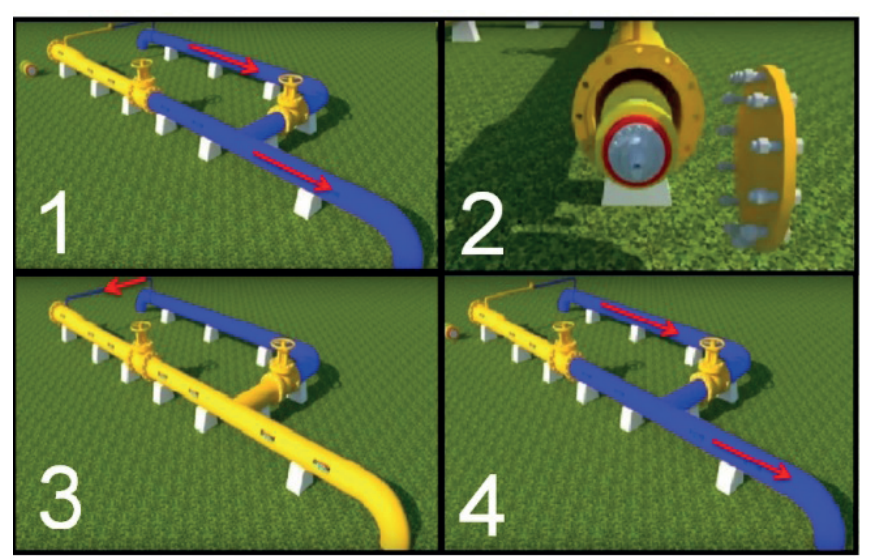

Fig. 2 Stages of placing the device Dacon [16]

Phase one: Standard pipeline operation. The medium flows in the direction indicated by the red arrow.

Phase two: Opening the hatch and inserting the diagnostic device.

Phase three: Closing the right large valve. The gas flow direction changes, which is indicated by the red arrow in Frame 3. The inflowing medium drives the inserted diagnostic device. After opening the left large valve, PIG leaves the driving station and moves with the medium.

Phase four: Opening the right valve to return to the initial flow [17].

\section{PARAMETERS OF THE NORD STREAM GAS PIPELINE}

Marine pipelines are extremely difficult to inspect. The Nord Stream gas pipeline has been laid at the bottom of the Baltic Sea and its task is to transport natural gas from Russia to Europe. This extremely large structure consists of two identical legs. The length of a single leg is $1224 \mathrm{~km}$, the inner diameter is $1.153 \mathrm{~m}$, and the thickness of walls varies from $27 \mathrm{~mm}$ to $41 \mathrm{~mm}$. When designing the pipeline, the pressure drop of the transported medium was taken into account which resulted in different wall thicknesses. The gas flow is generated by a set of compressors situated at the pipeline inlet. 
Laying the entire two-leg pipeline from Vyborg to Greifswald required the use of 202000 pipe segments of $24000 \mathrm{~kg}$ in weight each.

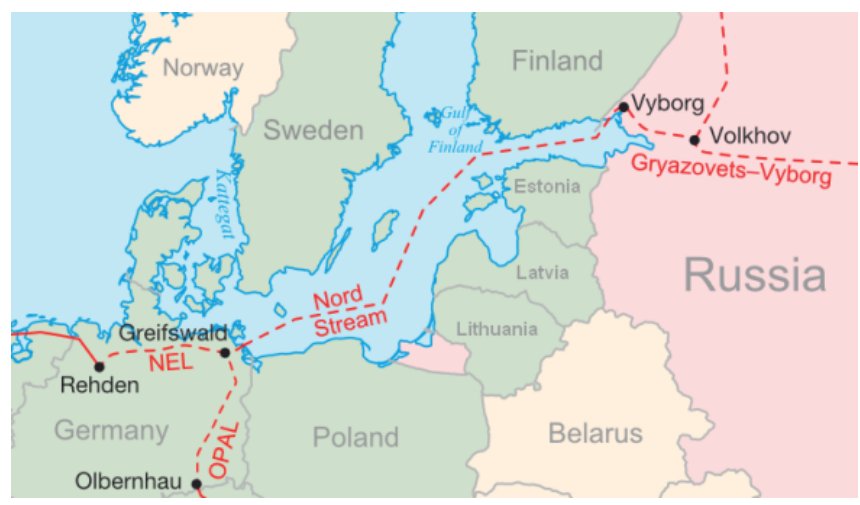

Fig. 3 The Nord Stream route [5]

Natural gas is a mixture of light saturated hydrocarbons, mainly methane. Since the molecular formula of the medium (natural gas) transported via the Nord Stream pipeline is a protected secret, the design calculations were to be preceded by molecular weight assessment of the flowing gas. The informational catalogue of DNV says that this is a dry gas with low sulphur content (95\% methane content, the minimum). Table 1 collates the molecular weight assessment according to PGNiG (Polish Petroleum and Gas Mining) for high-methane natural gas $[14,15]$.

\section{ECONOMIC DIAMETER}

The reliable input data used in economic diameter and gas flow velocity calculations corresponded to the calculation model described in [13].

Table 1. Assessing molecular weight of the deposit, acc. to [2]

\begin{tabular}{|c|c|c|c|c|c|}
\hline $\begin{array}{c}\text { Gas mixture } \\
\text { component }\end{array}$ & $\begin{array}{c}\text { Molecular weight ofgas } \\
\text { component } \\
\mathrm{M}_{\mathrm{cg}}\end{array}$ & $\begin{array}{c}\text { Relative density of gas } \\
\text { component } \\
\text { Gn } \\
{[-]}\end{array}$ & $\begin{array}{c}\text { Volumetric fraction } \\
\text { of gas component in } \\
\text { unit volume of mixture } \\
\text { (acc. to PGNiG) } \\
\mathrm{x}_{\mathrm{vg}}[-]\end{array}$ & $\begin{array}{c}\text { Molecular weight } \\
\text { fraction of gas } \\
\text { component in mixture } \\
\left(\mathrm{M}_{\mathrm{cg}}\right) \\
{[\mathrm{kg} / \mathrm{mol}]}\end{array}$ & $\begin{array}{c}\text { Relative density } \\
\text { fraction of gas } \\
\text { component in mixture } \\
\left(\mathrm{G}_{\mathrm{n}}\right)_{\mathrm{i}} \\
{[-]}\end{array}$ \\
\hline Methane & 16,14 & 0,56 & 0,978 & 15,785 & 0,545 \\
\hline Ethane & 30,07 & 1,04 & \multirow{3}{*}{0,010} & \multirow{3}{*}{1,323} & \multirow{3}{*}{0,046} \\
\hline Propane & 44,10 & 1,52 & & & \\
\hline Butane & 58,12 & 2,01 & & & \\
\hline Nitrogen & 28,01 & 0,97 & 0,010 & 0,280 & 0,010 \\
\hline Carbon dioxide & 44,01 & 1,52 & 0,002 & 0,088 & 0,003 \\
\hline
\end{tabular}

The economically justified inner diameter of the pipeline was calculated using formula (2) (with the temperature expressed in Kelvin degrees):

$\mathrm{D}_{\mathrm{ek}}{ }^{(\mathrm{g})}=0,397 \cdot 10^{-2} \cdot \mathrm{M}_{\mathrm{n}}{ }^{0,448} \cdot \mathrm{G}_{\mathrm{n}}{ }^{0,132} \cdot\left(\mu_{\mathrm{d}}{ }^{(\mathrm{g})}\right)^{0,025}\left(\frac{\mathrm{T} \cdot \mathrm{Z}}{\mathrm{P}}\right)^{0,316}$

where:

$\mathrm{D}_{\mathrm{ek}}{ }^{(\mathrm{g})}$ economically justified inner diameter of the pipeline $[\mathrm{m}]$

$M_{n} \quad$ volumetric gas flow rate (flow capacity of the pipeline) in normal conditions $\mathrm{T}_{\mathrm{n}}=273,15 \mathrm{~K}\left(\mathrm{t}_{\mathrm{n}}=0{ }^{\circ} \mathrm{C}\right), \mathrm{P}_{\mathrm{n}}=\mathrm{p}_{\mathrm{at}}$ $=101,325 \mathrm{kPa})\left[\mathrm{m}_{\mathrm{n}}^{3} / \mathrm{h}\right.$,

$\mathrm{G}_{\mathrm{n}} \quad$ relative gas density in normal conditions $\left(\mathrm{T}_{\mathrm{n}}=273,15 \mathrm{~K}\right.$ $\left.\left(\mathrm{t}_{\mathrm{n}}=0{ }^{\circ} \mathrm{C}\right), \mathrm{p}_{\mathrm{n}}=101,325 \mathrm{kPa}\right)(\mathrm{G}=1$ for air $)[-]$

$\mu_{d}{ }^{(g)}$ dynamic viscosity coefficient of gas $[\mathrm{MPa} \cdot \mathrm{s}]$,

$\mathrm{T}$ absolute gas temperature $[\mathrm{K}]$

$\mathrm{Z}$ gas compressibility ratio [-],

$\mathrm{P} \quad$ absolute gas pressure $[\mathrm{kPa}]$.

Based on the Nord Stream report by DNV, the outlet gas temperature was assumed constant and equal to $-1{ }^{\circ} \mathrm{C}$. Changing to Kelvin degrees gives:

$$
\mathbf{T}=273,15+(-1)=\mathbf{2 7 2}, \mathbf{1 5} \mathbf{K}
$$

The assumed pressure value needed in formula (1) is the arithmetic mean of the pipeline inlet and outlet pressures:

$$
\begin{aligned}
\mathbf{P}=\frac{\mathrm{p}_{1}+\mathrm{p}_{2}}{2}= & \frac{22+17,75}{2} \\
& =19,87 \mathrm{MPa} \\
& =\mathbf{1 9 8 7 0} \mathbf{~} \mathbf{P a}
\end{aligned}
$$

\footnotetext{
$G_{F}=M_{c g} \quad M_{c g} \quad$ gmolecular weight of gas $[\mathrm{kg} / \mathrm{kmol}]$,

$G_{n}=\frac{M_{c g}}{M_{c p}} M_{c p_{(m)}}^{M_{(m)}}$ molecular weight of air 28,964 [kg/kmol],
} 
The DNV report on Nord Stream construction says that the flow capacity of one pipeline leg in normal conditions is:

$$
\begin{aligned}
\mathbf{M}_{\mathbf{n}}=27,5\left[\mathrm{mld} \frac{\mathrm{m}_{\mathrm{n}}{ }^{3}}{\text { year }}\right] & \\
& =3,14\left[\mathrm{mln} \frac{\mathrm{m}_{\mathrm{n}}{ }^{3}}{\mathrm{~h}}\right] \\
& =\mathbf{3 1 3 9 2 6 9 , 4}\left[\frac{\mathbf{m}_{\mathbf{n}}{ }^{3}}{\mathbf{h}}\right]
\end{aligned}
$$

The relative density of gas in normal conditions is:

$$
\begin{array}{r}
\mathbf{G}_{\mathbf{n}}=\frac{\mathbf{M}_{\mathrm{cg}}}{\mathbf{M}_{\mathrm{cp}}}=\frac{17,476}{28,964}\left[\frac{\mathrm{kg} / \mathrm{kmol}}{\mathrm{kg} / \mathrm{kmol}}\right] \\
=\mathbf{0 , 6 0 3 [ - ]}
\end{array}
$$

The dynamic viscosity coefficient values are:

a) Beginning of the route: Vyborg. Maximal operating temperature:

$$
\begin{gathered}
\mathrm{T}_{\max }=40^{\circ} \mathrm{C}=313,15 \mathrm{~K} \\
\mu_{\mathrm{d}}(\mathrm{gt})=\mathbf{0}, 0119[\mathrm{mPa} \cdot \mathrm{s}] \\
\text { (the value read from Diagram } 1)
\end{gathered}
$$

b) End of route: Greifswald. Minimal operating temperature:

$$
\begin{aligned}
& \mathrm{T}_{\min }=-1^{\circ} \mathrm{C}=272,15 \mathrm{~K} \\
& \mu_{\mathrm{d}}{ }^{\mathrm{gt})}=\mathbf{0 , 0 1 0 7}[\mathrm{mPa} \cdot \mathrm{s}]
\end{aligned}
$$$$
\text { (the value read from Diagram 1) }
$$

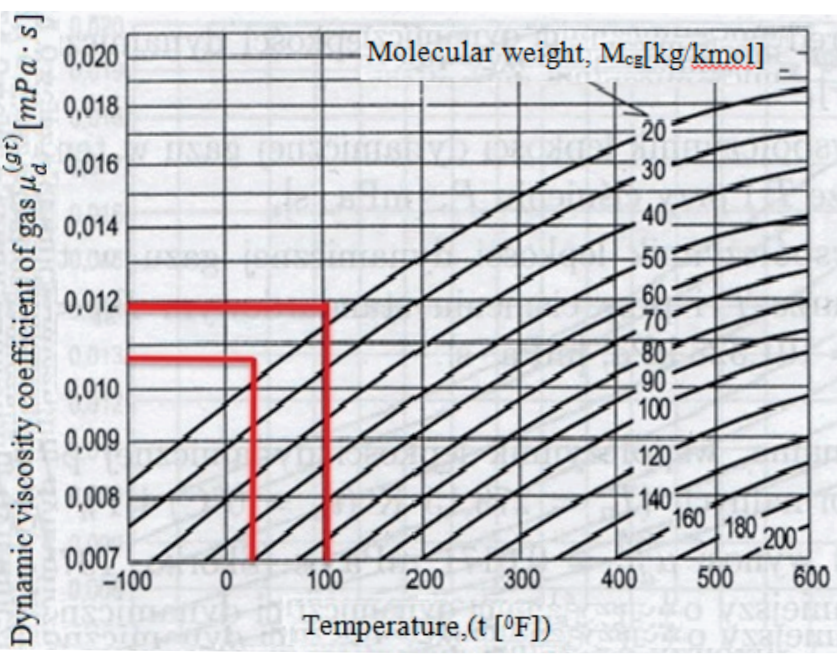

Diagram 1. Dynamic viscosity coefficient of gas vs. temperature and molecular weight of gas at atmospheric pressure [13].

To read the data from Diagram 1, the temperature was to be recalculated to Fahrenheit degrees: a) Inlet temperature:

$$
\left(40^{\circ} \mathrm{C} \cdot 1,8\right)+32=104^{\circ} \mathrm{F}
$$

b) Outlet temperature:

$$
\left(-1^{\circ} \mathrm{C} \cdot 1,8\right)+32=30,2^{\circ} \mathrm{F}
$$

The pseudocritical parameters of the gas mixture were calculated as:

$$
\begin{aligned}
& \mathrm{T}_{\mathrm{kr}}{ }^{(\mathrm{p})}=\sum_{\substack{\mathrm{i}=1 \\
\mathrm{n}_{\mathrm{g}}}}^{\mathrm{n}_{\mathrm{g}}}\left(\mathrm{T}_{\mathrm{kr}}\right)_{\mathrm{i}} \cdot\left(\mathrm{x}_{\mathrm{vg}}\right)_{\mathrm{i}} \\
& \mathrm{P}_{\mathrm{kr}}{ }^{(\mathrm{p})}=\sum_{\mathrm{i}=1}\left(\mathrm{P}_{\mathrm{kr}}\right)_{\mathrm{i}} \cdot\left(\mathrm{x}_{\mathrm{vg}}\right)_{\mathrm{i}}
\end{aligned}
$$

$\mathrm{T}_{\mathrm{kr}}$ (p) pseudocritical temperature of natural gas as the mixture of gas hydrocarbon substances $[\mathrm{K}]$,

$\mathrm{P}_{\mathrm{kr}}{ }^{\mathrm{p}}$ ) absolute pseudocritical pressure of natural gas as the mixture of gas hydrocarbon substances [Mpa]m

$\left(\mathrm{T}_{\mathrm{kr}}\right)_{\mathrm{i}}$ absolute critical temperature of $\mathrm{i}$-th natural gas component $[\mathrm{K}]$

$\left(\mathrm{P}_{\mathrm{kr}}\right)_{\mathrm{i}}$ absolute critical pressure of $\mathrm{i}$-th natural gas component, [MPa],

$\left(\mathrm{x}_{\mathrm{vg}}\right)_{\mathrm{i}}$ volumetric fraction of $\mathrm{i}$-th gas component in the mixture [-].

Critical parameters of particular natural gas components are given in Table 2.

Table 2. Selected physical parameters of hydrocarbons and other compounds [13]

\begin{tabular}{|c|c|c|}
\hline Name & Critical temperature & Critical pressure \\
\hline Methane & 191,11 & 4,64 \\
\hline Ethane & 305,0 & 4,88 \\
\hline Propane & 370,0 & 4,25 \\
\hline Butane & 425,56 & 3,80 \\
\hline Nitrogen & 126,11 & 3,39 \\
\hline Carbon dioxide & 304,44 & 7,40 \\
\hline
\end{tabular}

$$
\begin{aligned}
\mathrm{T}_{\mathrm{kr}}{ }^{(\mathrm{p})}=0,978 & \cdot 191,11+0,01 \cdot \frac{305+370+425,56}{3} \\
& +0,01 \cdot 126,11+0,002 \cdot 304,44 \\
& =\mathbf{1 9 2}, \mathbf{4 4}[\mathbf{K}]
\end{aligned}
$$

$$
\begin{aligned}
\mathrm{P}_{\mathrm{kr}}{ }^{(\mathrm{p})}= & 0,978 \cdot 4,64+0,01 \cdot \frac{4,88+4,25+3,80}{3}+ \\
& 0,01 \cdot 3,39+0,002 \cdot 7,40=\mathbf{4 , 6 3}[\mathrm{MPa}]
\end{aligned}
$$

The calculated values of the reduced temperature and pressure are:

$$
\mathrm{T}_{\mathrm{zr}}^{(\mathrm{p} 1)}=\frac{\mathrm{T}_{1}}{\mathrm{~T}_{\mathrm{kr}}(\mathrm{p})}=\frac{313,15}{192,44}=1,63
$$




$$
\mathrm{P}_{\mathrm{zr}}^{(\mathrm{p} 1)}=\frac{\mathrm{P}_{1}}{\mathrm{P}_{\mathrm{kr}}(\mathrm{p})}=\frac{22}{4,63}=4,75
$$

where:

$\mathrm{T}_{1} \quad$ inlet temperature,

$\mathrm{P}_{1} \quad$ inlet pressure,

$\mathrm{T}_{\mathrm{zr}}$ (pl) reduced pseudocritical temperature of natural gas at pipeline inlet [-]

$\mathrm{P}_{\mathrm{zr}}{ }^{(\mathrm{p} 1)}$ reduced pseudocritical pressure of natural gas at pipeline inlet [-]

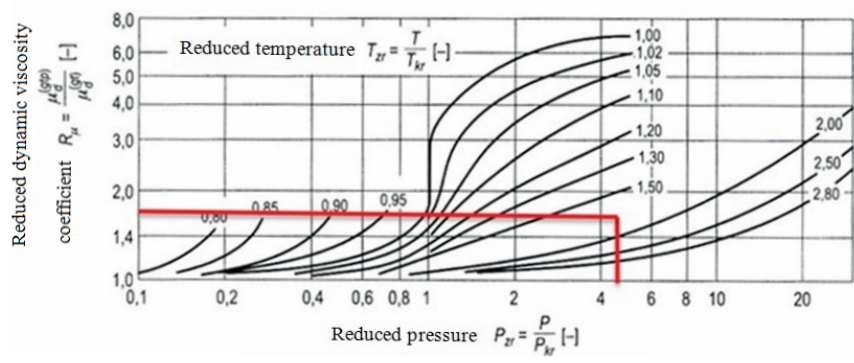

Diagram 2. Effect of the reduced pressure and reduced temperature on the dynamic viscosity coefficient of gas [13].

The read value of the dynamic viscosity coefficient of gas is:

$$
R_{\mu}=\mu_{d}^{(g t p)} / \mu_{d}^{(g t)}=\mathbf{1 , 6}
$$

where:

$\mu_{\mathrm{d}}{ }^{\text {(gtp) }}$ dynamic viscosity coefficient of gas at temperature $\mathrm{T}$ and pressure $\mathrm{P}[\mathrm{MPa} \cdot \mathrm{s}]$

$\mu_{\mathrm{d}}{ }^{(\mathrm{gt})}$ dynamic viscosity coefficient of gas at temperature T and standard pressure $\mathrm{P}_{\text {st }}=101325[\mathrm{mPa} \cdot \mathrm{s}]$

The viscosity coefficient in flow conditions was assessed as: $\boldsymbol{\mu}_{\mathrm{d}}^{(\mathrm{g})} \equiv \mu_{\mathrm{d}}{ }^{(\mathrm{gtp})}=\mathrm{R}_{\mu} \cdot \mu_{\mathrm{d}}{ }^{(\mathrm{gt})}=1,6 \cdot \mu_{\mathrm{d}}{ }^{(\mathrm{gt})}=1,6 \cdot 0,0119=\mathbf{0 , 0 1 9 0 4}[\mathrm{mPa} \cdot \mathbf{s}]$

The compressibility ratio of the real gas was read from Diagram 3.

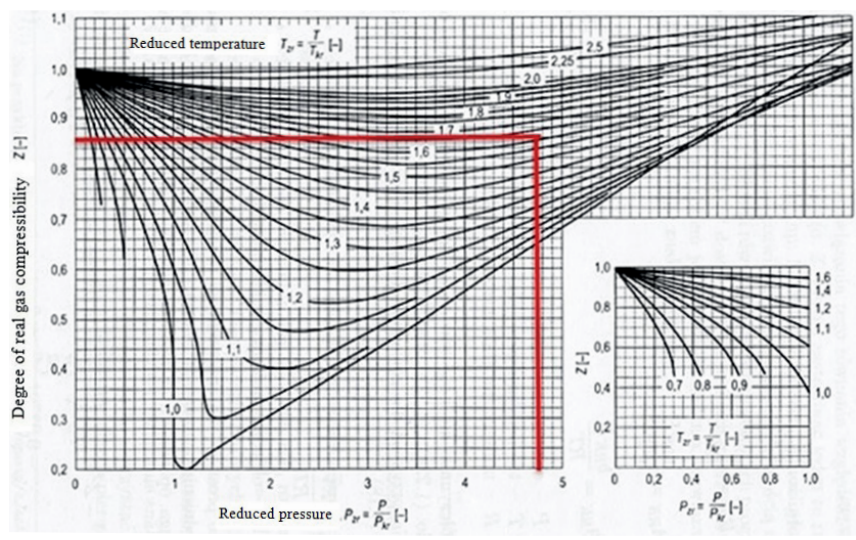

Diagram 3. Gas compressibility ratio vs. reduced pressure and temperature [13].

The read value: $\mathbf{Z}=\mathbf{0 , 8 5 5}$

The economic diameter of the pipeline was calculated from the formula:

$$
\begin{aligned}
& D_{e k}{ }^{(g)}=0,397 \cdot 10^{-2} \cdot M_{n}{ }^{0,448} \cdot G_{n}{ }^{0,132} \cdot\left(\mu_{d}{ }^{(g)}\right)^{0,025} \cdot\left(\frac{T \cdot Z}{P}\right)^{0,316} \\
& \begin{aligned}
\mathrm{D}_{\text {ek }}{ }^{(\mathrm{g})}=0,397 \cdot 10^{-2} \cdot 3139269,4^{0,448} \cdot 0,603^{0,132} \cdot(0,01904)^{0,025} \cdot\left(\frac{272,15 \cdot 0,855}{19870}\right)^{0,316} \\
=\mathbf{0}, \mathbf{6 7} \mathbf{~} \mathbf{~} \approx \mathbf{0}, \mathbf{7} \mathbf{~}
\end{aligned}
\end{aligned}
$$

Unlike liquid media, the transported natural gas is compressible, therefore of high importance is placing in the above formula real temperature and pressure values, as they are the only parameters reflecting real flow conditions in the pipeline, while the remaining parameters refer to normal conditions, i.e.: $\left(\mathrm{T}_{\mathrm{n}}=273,15 \mathrm{~K}\left(\mathrm{t}_{\mathrm{n}}=0^{\circ} \mathrm{C}\right), \mathrm{P}_{\mathrm{n}}=\mathrm{p}_{\mathrm{at}}=\right.$ $101,325 \mathrm{kPa}$ ). The volume of the transported gas depends on its temperatre and pressure, therefore normal calculating conditions were introduced to standardise particular parameters and thus provide opportunities for comparing, for instance, volumetric flow rates or densities with other gas media. The inner diameter of the Nord Stream gas pipeline is $1,153 \mathrm{~m}$, while the calculated economic diameter was only $0,67 \mathrm{~m}$. That means that the real diameter is larger by $41,9 \%$ than the economic diameter. This discrepancy can result from calculating the economic diameter with the aid of the formula created based on the steel pipeline production costs recorded in the USA in 1975. For gas pipelines produced in those years the formula worked very well, with the difference between real and economic diameter not exceeding a few percent. The author of [13] quotes that the steel pipe production cost increased by $5 \%$ until 1998, while the cost of electrical energy, taken into account in the formula, increased by $10 \%$. The Nord Stream pipeline is an undersea pipeline, which has been built since 2010 . The production costs were extremely high, and the predicted operation time is equal to 50 years. In case of such investment projects, in the initial phase the economically justified diameter is calculated based on material costs, operating time, etc., and only in the next phase the operating diameter is calculated. These two diameter values can differ.

\section{GAS FLOW VELOCITY IN NORD STREAM PIPELINE}

The medium flow velocity in the Nord Stream gas pipeline was calculated from formula (9) [2]:

$$
v_{g}=0,131 \cdot 10^{-3} \cdot\left(\frac{M_{n} \cdot T \cdot Z}{P \cdot\left(D_{w}^{(g)}\right)^{2}}\right)
$$

where:

$\mathrm{v}_{\mathrm{g}}$ natural gas flow velocity $[\mathrm{m} / \mathrm{s}]$,

$\stackrel{\mathrm{g}}{\mathrm{m}}_{\mathrm{n}} \quad$ volumetric gas flow rate $\left[\mathrm{m}^{3} / \mathrm{h}\right]$,

$\mathrm{T}^{\mathrm{n}}$ absolute gas temperature $[\mathrm{K}]$,

$\mathrm{Z}$ gas compressibility ratio [-],

$\mathrm{P}$ absolute gas pressure [kPa],

$\mathrm{D}_{\mathrm{w}}^{(\mathrm{g})} \quad$ inner diameter of pipeline [m]. 


$$
\begin{gathered}
\mathbf{v}_{\mathbf{g}}=0,131 \cdot 10^{-3} \cdot\left(\frac{\mathrm{M}_{\mathrm{n}} \cdot \mathrm{T} \cdot \mathrm{Z}}{\mathrm{P} \cdot\left(\mathrm{D}_{\mathrm{w}}^{(\mathrm{g})}\right)^{2}}\right)= \\
0,131 \cdot 10^{-3} \cdot\left(\frac{3139269,4 \cdot 272,15 \cdot 0,855}{19,875 \cdot(1,153)^{2}}\right)=\mathbf{3 , 6 2}\left[\frac{\mathbf{m}}{\mathbf{s}}\right]
\end{gathered}
$$

The diagnostic PIG is assumed to move with the same velocity as that of the transported medium (here: natural gas).

\section{ELEMENTS OF DESIGNED DEVICE}

The diameter of the designed device is equal to the inner diameter of the Nord Stream pipeline. This condition is essential, as the measuring, cleaning and driving elements should be in tight contact with the pipeline wall to work properly. The length of the device mainly results from space requirements for the container with electronics and the battery for supplying measuring instruments during the inspection. The calculated capacity of the battery is $28,8 \mathrm{kWh}$, and its weight is $274,28 \mathrm{~kg}$. The battery consists of 4 packages of 178 small batteries and 1 package of 67 small batteries. The energy stored in the battery is sufficient for 4 days of device operation.

The designed smart device consists of four indispensable sections.

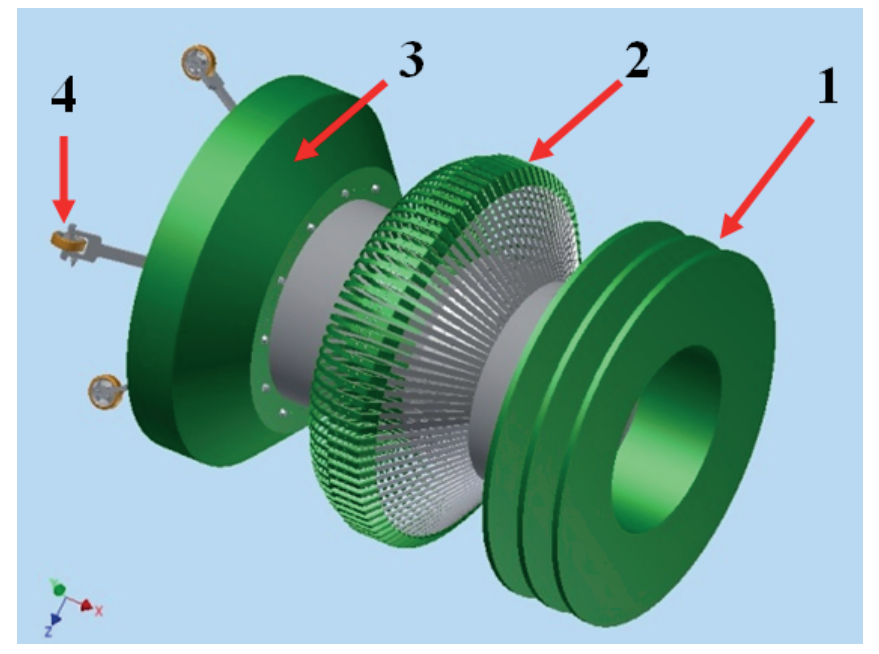

Fig. 4. Complete diagnostic device [10]

1 - cleaning seal, 2 - measuring element, 3 -driving seal, 4 -distance gauge.

Functions performed by the device include:

a) pipeline cleaning from dirt,

b) measuring the covered distance,

c) detecting gas pipeline diameter changes.

Planned tasks:

a) PIG is expected to perform internal inspection of the pipeline for which it was built. The inspection consists in detecting material losses, cracks, and/or tarnishes. Fast detection of defects will make it possible to avoid gas pipeline unsealing and the resultant ecological disaster, b) Overall diagnostics and precise location of places which need intervention,

c) Location of hydrate concentration places.

The number of functions and tasks directly affects the number of segments used in the device. The first element is the cleaning seal. It is situated in the front part of the vehicle and its task is to collect tarnishes and dirt remaining after the previous cleaning. This element consists of three discs, which makes its structure more rigid and the cleaning process more precise. The discs have empty space in the centre, to limit the mass of the device. Moreover, this space plays the role of a container for collected dirt, thanks to which the wedges of the cleaning discs will not get clogged. The second element of the device is the section with the glued strain gauge bridges. It detects dents, lumpy tarnish and dirt on pipeline walls. The perimeter of this section contains 90 measuring loop-like elements mounted every 4 degrees around the perimeter of the device casing.

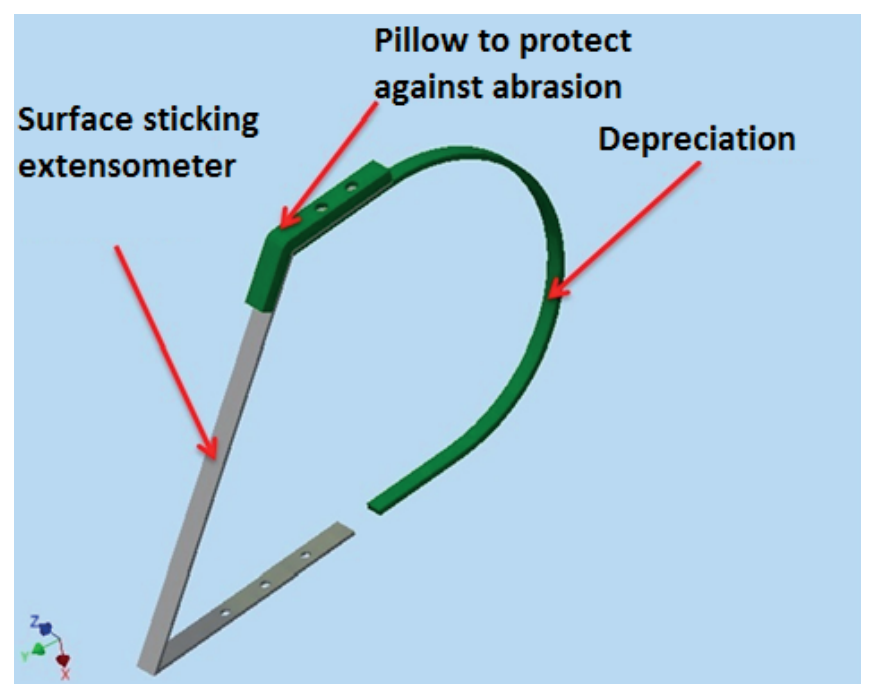

Fig. 5 Measuring element [10]

The error of perimeter imperfection detection was calculated in the following way:

Length along perimeter:

$$
\begin{gathered}
L=2 \cdot \pi \cdot r \\
L=2 \cdot \pi \cdot 576,5=3622,26 \mathrm{~mm}
\end{gathered}
$$

where:

$r$ inner diameter of pipeline,

90 number of measuring elements,

$20 \mathrm{~mm}$ width of measuring element,

$$
\begin{gathered}
90 \cdot 20=1800 \mathrm{~mm} \\
3622,26-1800=1822,26 \mathrm{~mm} \\
\frac{1822,26}{90}=\mathbf{2 0 , 2 4} \mathbf{~ m m}
\end{gathered}
$$

The width of the gaps between the measuring elements is equal to $20,24 \mathrm{~mm}$. This accuracy is sufficient for detecting 
significant defects. A strain gauge bridge is glued to each measuring element. Proper selection and arrangement of these bridges enables to detect pipeline diameter changes, and further precise analysis of the detected pits, tarnishes and corrosion, and the current condition of welded joints. High sensitivity of strain gauge bridges provides opportunities for precise tracking of changes on pipeline walls [11]. The third part of the inspection device is the sealing element. It prevents the natural gas from seeping behind the element, thanks to which the entire mass of gas presses into the front wall of the inspection device and moves it downstream. The fourth section of the device consists of rolls, mounted every 90 degrees and fitting to the gas pipeline walls. The motion of the designed device makes the rolls rotate, and thus measure the distance covered by the PIG. Figure 6 shows the positions of the measuring rolls and inductive sensors.

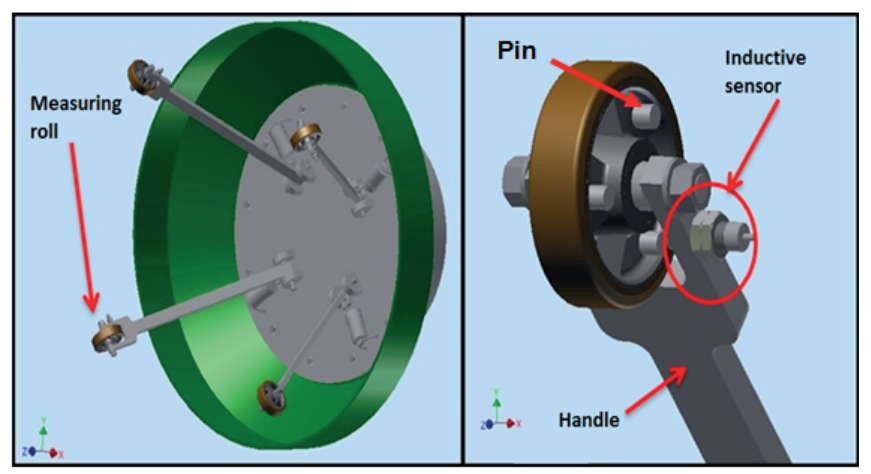

Fig. 6 The distance meter [10].

The inductive sensor is screwed into the fork (measuring roll handle), at a distance of $4 \mathrm{~mm}$ from the pin. This distance is sufficient for safe counting of the number of sensor's meetings with the pin. Each disc with the rolls has 3 metal pins, fastened every 120 degrees. Counting the average number of revolutions recorded by 4 rolls makes it possible to calculate precisely the current position of the device. The device project assumes installing the sensor IS-12-H1-S1 made by Simex [12]. The measuring rolls work in difficult conditions and they should be reliable and resistant to damages. When selecting this element, it is advisable to take into account rolls used in forklift trucks. They reveal good adhesion and low abrasibility, and are offered in many sizes on the marker. The rolls selected for the device project were made by Elesa-Ganter [20]. Along with the measuring elements, the potentiometer is used for detecting pipeline diameter changes (dents, welds, thick tarnish). However, in this case it also plays another function. Each diameter change may cause vibrations or jumps of the rolls measuring the distance covered by the device, which may lead to the accumulation of errors in device position estimation. In this situation the potentiometer helps to identify and properly correct the accumulated position errors [6,7]. The inspection device is also equipped with a spring shock absorber, the task of which is to keep four forks in such a position that all measuring rolls are pressed to pipeline walls. Proper tension of the spring keeps the forks in correct positions, and thus makes the rolls be in tight contact with the walls. The designed shock absorber consists of the spring, a piston and a mandrel screwed into it. Its casing is assumed to be airtight, to protect the shock absorber interior from fouling. The number of movements of the PIG device is limited, as the pipeline in which it moves constitutes a sort of track for it. The device is solely driven by the medium flowing in the pipeline. The only independent movement which PIG can make is changing the rotation angle around its own axis, which is facilitated by axial symmetry of the device along its length. Theoretically, the rotational position of the inspection probe can be measured using oscillatory or liquid inclinometers. They work well on slow ships, where the reading of the measuring element is obtained directly. Nevertheless, for higher speeds and accuracies, other types of inclinometers are recommended. A place for this measuring instrument is provided in the airtight container for electronics. The project assumes the use of one-dimensional inclinometer IS1SP360-BL produced by WObit. Its measuring range is up to 360 degrees, and its accuracy is very high. The sensor is factory calibrated. According to the producer, it works very well in difficult conditions. The natural gas pressure in the Nord Stream pipeline is sufficiently high to introduce the PIG device for inspection purposes. However, to move properly, the device should have a relevant "driving surface", which means that the gas flowing in the pipeline should press the inspection device evenly and thus generate a relevant driving force. The speed of motion of the inspection device is assumed to be approximately equal to that of the flowing medium. The role of driving surface is played in the device by the driving seal. It is in permanent tight contact with pipeline walls, thus blocking the pipeline cross section and generating the maximum driving force. A set of three discs is also mounted in the front part of the device body. They have two tasks to perform, which are driving and cleaning. Since the gas pipeline is not as heavily fouled as the oil pipeline, a decision was made to resign from installing cleaning brushes on the device body.

\section{SUMMARY}

The designed inspection device consists of three decouplable segments, which means that it can be disassembled into three main parts: stern part with the driving seal and instrumentation, middle part with the measuring elements, and front part with a set of polyurethane discs. The three-part structure was designed with an intention of easy replacement of individual elements. The system of measuring elements distributed around the device perimeter has a wide inspection range. Substantial damages should be detected. Indeed, the measuring elements will be subject to wear, which first of all means abrasion of measuring element shields. When their thickness decreases to the height of the countersunk head of the fastening rivet, the polyurethane shield is to be replaced. The external potentiometer is also exposed to quick damage. Acting within a small angular range, it is exposed 
to so-called "erasing wear". That is why of high importance is visual examination of the device after each inspection operation. As for the distance meter, its fastening on the steel fork can be replaced by laminate elements, which reveal high strength and material elasticity. The designed inspection device had no assumed weight limits, therefore the applied mechanical structure of the distance meter support has no contraindications concerning its use. What is more, it is relatively simple and cheap to manufacture. When selecting the inclinometer, of high importance was to know its structure and principle of operation. The pipeline will be a sort of screen for the inclinometer, therefore its operation should base on the acceleration due to gravity. The inclinometer selected for the project is reliable and relatively small, and has a wide operating range.

Based on simple solutions, a device was designed which performs cleaning and inspection tasks in functional and reliable way. Its servicing should not pose a problem, as all elements are easily replaceable. Moreover, by using smaller or larger sealing discs and different sizes of measuring element shock absorbers, the designed PIG device can be easily adapted for operation in pipelines with other inner diameters. Here, the only limitation is the diameter of the container for electronics and battery.

\section{BIBLIOGRAPHY:}

1. http://osfm.fire.ca.gov/pipeline/pdf/conferece/ inlineinspection.pdf,

2. http://www.peg-germany.de/en/products/pipelineproducts/pipeline-spheres.html,

3. Rowiński L.: Deep-water vehicles: structure and equipment (in Polish), WiB Gdansk 2008,

4. http://www.inlineservices.com/pipeline-pig-brushes,

5. https://deutsch.rt.com/wirtschaft/49870-ostsee-pipelinenord-stream-2-trotz-sanktionen-gebaut-russlanddeutschland/

6. http://rab.ict.pwr.wroc.pl/ arent/rr/mpr/czujniki2.html,

7. http://www.tme.eu/pl/details/cw-18-10k-p6/ potencjometry-jednoobrotowe-cermetowe/telpod/,

8. Hupka J.: Pipeline operation related technical problems (in Polish), Department of Chemical Technology, Faculty of Chemistry, Gdansk University of Technology.

9. Gerwatowski D.: Linear flow resistance coefficient vs. system flow capacity, with the Yamal pipeline as an example of transit pipeline system (in Polish), EuRoPol GAZ SA, Warsaw.

10. Figures from Inventor programme.
11. http://automatykab2b.pl/technika/3095-czujnikitensometryczne-czyli-co-o-pomiarach-siy-powinienwiedzie-automatyk\#.VmstudLhC00,

12. http://www.simex.pl/produkt/3313,

13. Magda W.: Marine pipelines (in Polish), Wydawnictwo techniczne, Warsaw 2004.

14. Nord Stream - Espoo Report, https://www.nord-stream. com

15. http://www.nord-stream.com/

16. https://www.youtube.com/watch?v=55wa_CvA7UA,

17. http://www.bc.pollub.pl/Content/254/korozja.pdf

18. http://www.tdwilliamson.com/SolutionsEngine?conditio $\mathrm{n}=99$ \&industry $=9$,

19. http://www.rosen-group.com/global/solutions/pipelinesystems.html,

20. http://www.elesa-ganter.com/pl/28/sp/9563/4/120/kolaz-nalewanym-wie\%C5\%84cem-poliuretanowym/re.f5/ eg/,

\section{CONTACT WITH AUTHOR}

\section{Danuta Warnke}

e-mail:danwarnk@pg.gda.pl

Gdansk University of Technology

ul. Narutowicza 11/12

80-233 Gdansk 